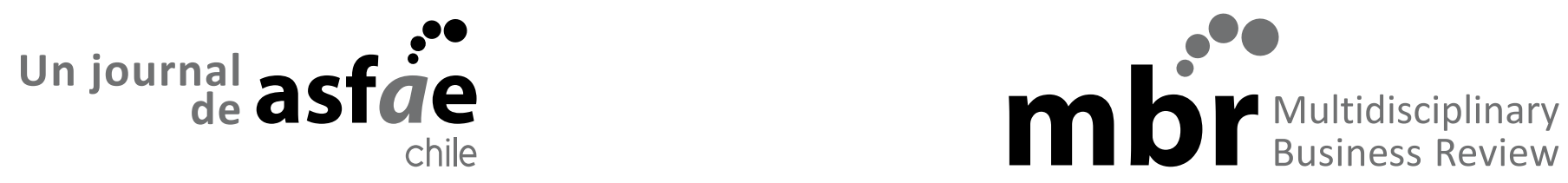

\title{
EL MOBBING: UN ESTUDIO EN EL SECTOR EDUCATIVO ESPAÑOL
}

\author{
MOBBING: A STUDY IN THE SPANISH EDUCATIONAL SECTOR
}

\author{
Ana María Aguilera Luque \\ Clasificación: empírico - investigación \\ Recibido: 8-junio-2020 / Revisado: 24-noviembre-2020 / Aceptado: 27-enero-2021
}

\section{Resumen}

Este artículo se propone analizar el mobbing o acoso en el trabajo y sus relaciones con diversas variables relacionadas con la persona, las interacciones en el entorno de trabajo y el comportamiento organizacional que pueden ser antecedentes o consecuentes del acoso en el sector educacional. La metodología es correlacional y transversal, y emplearon escalas autoinforme en una muestra de 103 participantes. Algunas de las relaciones y los efectos propuestos se han confirmado en este estudio. A través del análisis de mediación se confirma el efecto directo de la tolerancia organizacional sobre el mobbing, que puede explicar hasta el $20 \%$ de la varianza en la escala NAQ, así como las variables nivel de estudios y género. Igualmente, se confirma el efecto directo del mobbing en la salud. El modelo propuesto explica el $25 \%$ de la varianza en el autoinforme de salud. Es destacable la alta incidencia de acoso psicológico en esta muestra. Este estudio busca proporcionar información que ayude a las organizaciones a desarrollar políticas orientadas a mejorar el lugar de trabajo en materia de acoso.

Palabras clave: mobbing, bullying, acoso, acoso laboral, tolerancia organizacional.

\begin{abstract}
This study aims to analyze mobbing or harassment at work, and its relations with various variables related to the person, interactions in labor settings, and organizational behavior that can be predictors or consequents of harassment in the educational sector. The methodology is correlational and cross-sectional, using self-report type scales in a sample of 103 participants. Some of the proposed relations and effects have been confirmed in this study. Through SEM-mediation analysis, the direct effect of organizational tolerance on mobbing is confirmed, which can explain up to $20 \%$ of the variance in NAQ scale, as well as the variables, level of studies and genre. Equally, the effect of mobbing on health is confirmed. The proposed model explains $25 \%$ of the variance in self-report of health. It is remarkable the high incidence of mobbing in this sample. This study tries to provide information that helps organizations develop policies oriented to improve the workplace regarding mobbing.
\end{abstract}

Keywords: mobbing, bullying, harassment, workplace harassment, organizational tolerance.

a Tecnológico de Monterrey. Puebla, México. E-mail: ana.aguilera@tec.mx 


\section{Introducción}

Las conductas incívicas y nocivas constituyen una realidad frecuente en el mundo laboral y generan efectos adversos en las personas y en las organizaciones (Andersson y Pearson, 1999; Cortina, 2008; Einarsen et al., 2003; Hirigoyen, 1998; Leymann, 1996; Topa, Depolo y Morales, 2007). Uno de los componentes clave de los ambientes laborales tóxicos es el acoso laboral, también denominado acoso moral en el trabajo (Hirigoyen, 1998), mobbing (Leymann, 1990; 1996; Zapf, 1999), bullying (Rayner y Cooper, 1997), psicoterror (Leymann, 1996) u hostigamiento psicológico en el trabajo (Fornés et al., 2008).

El mobbing o acoso laboral se considera un proceso de agresión sistemática y repetida por parte de una persona o grupo hacia un compañero, subordinado o superior, y que tiene lugar en el contexto de trabajo (Einarsen, 2000; Leymann, 1990; 1996). La mayoría de estudios lo consideran un fenómeno universal en el que se presenta: a) reiteración de actos negativos y hostiles hacia una persona en su trabajo y b) prolongados en el tiempo y con carácter de continuidad; c) incapacidad de la víctima para defenderse y d) gravedad de las consecuencias indeseables sobre la víctima (Topa et al., 2007); e) intencionalidad del acosador (una o varias personas) de carácter instrumental o de carácter finalista, y f) desequilibrio de poder (real o percibido) de las partes implicadas (Einarsen, 2000).

El mobbing es un riesgo sociolaboral, un fenómeno multifacético (Zapf, 1999) abordable desde diversas perspectivas teóricas (Einarsen et al., 2003). Es un proceso gradual (Einarsen, 2000; Leymann, 1990) que evoluciona desde etapas iniciales, en las que las conductas hostiles son sutiles y pasan desapercibidas a los observadores, hasta fases más activas en las que, por la implicación de superiores y compañeros, se puede llegar a responsabilizar a la víctima de la situación que padece (Einarsen, 1999).

El acoso en el trabajo se considera uno de los estresores sociales más importantes (Leymann, 1996; Zapf, 1999), y, dado que el estrés se relaciona con problemas de salud física y psíquica (Nielsen et al., 2013; Topa et al., 2007; Topa et al., 2009), puede deducirse la gravedad de este fenómeno para las personas, las organizaciones y la sociedad en su conjunto. Precisamente, el estrés - y este en sus formas más extremas, como, por ejemplo, el burnout - ha sido un tema de investigación dentro del sector educativo y de la profesión docente (Farber y Miller, 1981).

En cuanto a su incidencia, la última Encuesta Europea Sobre Condiciones de Trabajo-2015 (Parent-Thirion et al., 2016) indica que el $16 \%$ de la población europea está sujeta a una conducta social adversa en el lugar de trabajo, y es España el país con menor incidencia (10\%). Se encuentran estudios que indican que el $70 \%$ de trabajadores ha experimentado algún tipo de acoso en el trabajo de forma directa o indirecta, como observadores o testigos de estas conductas (Einarsen et al., 2003).

\section{La agresión en el estudio del mobbing}

El mobbing comporta agresión, entendida como cualquier forma de conducta intencionada dirigida a dañar o injuriar a otro ser vivo que está motivado a evitar tal trato (Baron y Richardson, 1994). Agresión es también cualquier intento de difamación o de provocar temor a una víctima. La intencionalidad de daño ha supuesto que algunos autores describan al acosador como psicópata organizacional (Villagrán et al., 2017) o ser perverso (Hirigoyen, 1998).

Una de las teorías explicativas de la conducta agresiva es la del aprendizaje social que propone que esta conducta se adquiere, mantiene y desarrolla mediante el modelado y la imitación (Bandura et al., 1963). Por esta razón, la cultura es un predictor de máxima importancia de la conducta agresiva y se considera un mecanismo regulador de la agresión en las relaciones interpersonales y sociales. En el ámbito laboral, puede entenderse que los comportamientos de agresión estarían regulados por la cultura organizacional y/o la del grupo en el que se integre el individuo. Esto se relaciona con la identidad social (Tajfel, 1970; 1974; Tajfel y Turner, 1979; Turner y Brown, 1978) y con la tolerancia organizacional (OT) a las conductas de acoso (Andersson y Pearson, 1999; Cortina, 2008; 2015; Einarsen, 2000; Pérez-Larrazabal, 2016; Pérez-Larrazabal y Topa, 2016; Pérez-Larrazabal, Lopez de la Llave y Topa, 2019).

\section{Potenciales antecedentes del mobbing}

Dentro de los factores demográficos, algunos estudios apuntan a una mayor frecuencia de acoso laboral a mujeres y jóvenes, mientras que la antigüedad del trabajador parece correlacionar negativamente con el acoso (Topa et al., 2007). El primer objetivo del presente trabajo se centra en estos aspectos y trata de verificar dichos hallazgos en la población de trabajadores de la educación españoles, estableciéndose cuatro hipótesis de incidencia de acoso según las siguientes variables sociodemográficas:

- H1: mayor incidencia de acoso en la población femenina. 
- H2: mayor incidendia de acoso en los trabajadores más jóvenes.

- H3: la antigüedad en la organización amortigua las conductas de acoso.

- H4: un mayor nivel de estudios disminuye la incidencia de acoso.

En cuanto a las características personales y de interacción en el contexto laboral, la capacidad de ajuste o adaptabilidad de la persona es un factor asociado a los procesos de discriminación y acoso laboral (Cortina, 2008). Esta relación puede darse en dos sentidos distintos: a) el trabajador puede convertirse en diana de dinámicas nocivas por no ajustarse a las normas del grupo/organización o, si el ajuste se produce en un entorno tolerante y promotor de dichas conductas, b) puede convertirse en instigador o consentidor de dinámicas de acoso.

Estudios previos han encontrado una relación negativa entra la adaptabilidad del individuo a su entorno y las conductas contrapoducentes en el trabajo, así como un efecto moderador de aquella en la relación entre tolerancia organizacional (OT) y acoso (Pérez-Larrazabal, 2016). Con el propósito de comprobar su potencial relación negativa con las conductas de acoso, se plantea la siguiente hipótesis:

- H5: la adaptabilidad del empleado disminuye la probabilidad de acoso en el trabajo.

Todavía en el eje de la relaciones personales, dos de los enfoques teóricos en los que se apoyan diversos estudios sobre acoso son la teoría de la identidad social (TIS) (Tajfel, 1970; 1974; Tajfel y Turner, 1979; Turner y Brown, 1978) y la teoría de la autocategorización del yo (TAC) (Turner, 1985; Turner et al., 1987), así como en la relación de ambas con el sentimiento de pertenencia y en los efectos de dicha identificación y/o del apoyo social percibido. La identificación es un fenómeno psicológico que influye en las actitudes y los comportamientos de las personas. Diversos estudios han demostrado que la identificación es un buen predictor de diversos fenómenos tales como la satisfacción laboral, la implicación en el trabajo, la motivación, el rendimiento y las conductas de ciudadanía, entre otros (Topa et al., 2008).

En relación con la identificación, se ha encontrado que unas relaciones interpersonales insatisfactorias y destructivas pueden actuar como antecedentes en la definición y expresión del acoso laboral. Este aspecto adquiere gran importancia en las organizaciones muy reglamentadas, con fuertes vínculos e identidades compartidas y una escasa tolerancia a la diversidad (Segurado et al., 2008). Asimismo, la identificación social parece ser uno de los antecedentes clave en la aparición del apoyo social y este, a su vez, parece ser un importante amortiguador de los efectos del acoso, al actuar como mediador entre la identificación y el acoso (Topa et al., 2009). Sobre la base de estos supuestos se plantea la siguiente hipótesis:

- H6: las distintas dimensiones de la identificación social en el trabajo (organizacional, grupal y con la profesión), correlacionan negativamente con las conductas de mobbing percibido.

En el tercer eje causal, referido a las dimensiones organizacionales, un factor relativo a la estructura es el tamaño de la organización, para el cual se han planteado distintos supuestos. Una organización de mayor tamaño, tal vez, tenga mejor reguladas sus políticas contra el acoso, así como una mayor actividad sindical de control sobre este tipo de fenómenos, lo que podría dismininuir su incidencia. Una perspectiva alternativa sería que al tener un mayor número de empleados y ser, por tanto, más difícil observar las relaciones que se producen, la incidencia de mobbing puede ser mayor. Desconociendo de antemano el posible sentido de la relación, se plantea la siguiente hipótesis con carácter exploratorio:

- H7: existe una relación entre la aparición de conductas de acoso y el tamaño de la organización en cuanto a su número de empleados.

Otros estudios indican que las características del contrato laboral también se relacionan con el acoso. Se ha encontrado que las personas contratadas a tiempo parcial, con contratos temporales o subcontratados, se relacionan con mayor inseguridad en el trabajo, percibida o real, y esta situación puede actuar como antecedente de la conducta de acoso (Björkqvist et al., 1994). En este sentido, se plantea la siguiente hipótesis:

- H8: existe una relación positiva entre la aparición de conductas de acoso y la situación de contratación a tiempo parcial.

Respecto a los factores relativos al ambiente de trabajo y al clima organizacional, diversos estudios los consideran los predictores más eficaces de las conductas de acoso (Cortina, 2008; 2016; Einarsen, 2000; Leymann, 1990; Salin, 2003; Zapf, 1999). Cortina (2016) incide en que el factor predictor organizacional del acoso, tanto para predecir la ocurrencia como para predecir el daño que provocará, es el clima organizacional. El metaálisis de Topa y sus colegas (Topa et al., 2007) apoya este supuesto, de modo que coindicen en que dichos factores, fudamentalmente, son: 
a) conflicto y ambigüedad de rol, b) exceso de demandas laborales, c) el grado de autonomía en el trabajo y d) el apoyo social.

Relacionado con el clima organizacional y con miras a delimitar el origen y la progresión de estas conductas y su intencionalidad, Andersson y Pearson (1999) proponen la incivilidad como precursora y definen la civilidad en el lugar de trabajo como un comportamiento que ayuda a preservar las normas de mutuo respeto en el entorno laboral (p. 455). Las conductas que se enmarcan como incívicas serían formas menores de maltrato que rompen las normas de respeto mutuo y generan percepciones de injusticia organizacional. Esto puede generar una espiral de agresividad posterior. El contexto es la variable influyente en estas conductas, de manera que se proponen tres aspectos relacionados con ellas: la política antidiscriminatoria (o su ausencia), la actitud de los líderes (Padilla et al., 2007) y las normas del grupo o equipo de trabajo (Cortina 2008), lo que se relacionaría con la tolerancia organizacional (OT) ante las conductas de acoso (PérezLarrazabal, 2016; Pérez-Larrazabal y Topa, 2016; PérezLarrazabal et al., 2019; Richman et al., 2004).

Aunque diversos estudios teóricos apuntan a la influencia de la OT en las conductas de acoso, existe poca investigación empírica sobre esta cuestión. Perez-Larrazabal et al. (2019), continuando con el trabajo de Pérez-Larrazabal (2016), incrementan el corpus de conocimiento al presentar su escala POT para medir la OT, así como la validación de esta en una muestra de trabajadores. En su investigación encuentran que la tolerancia organizacional la componen cinco dimensiones o factores: formación, evaluación, ética, coherencia y promoción.

Ahondando en estas dimensiones, los autores proponen que una organización que facilita un alto grado de formación sobre conductas de acoso, evalúa las acciones que se han adoptado para detectarlo, denunciarlo y contenerlo, promueve acciones y facilita información sobre los resultados, actúa de forma ética promoviendo políticas de igualdad e inclusión y actúa con coherencia respecto a lo que declara, conseguirá minimizar la ocurrencia de acoso y frenar rápidamente su escalada y sus efectos, en caso de que pudiera manifestarse.

En el contexto de este estudio, por tanto, la tolerancia organizacional se entiende como la permisividad, desatención y/o negligencia que una organización puede presentar ante los actos incívicos que en ella pueden darse (Einarsen, 2000; Pérez-Larrazabal, 2016; Richman et al. 2004). Pérez-Larrazabal puntualiza que dicha permisividad puede ser intencionada o no y que la actitud adoptada por la organización puede superar la permisividad respecto al acoso para convertirse en connivencia, de forma que la propia organización estaría promoviendo el acoso, más allá de consentirlo. En este sentido se propone la siguiente hipótesis:

- H9: el grado de tolerancia de la organización respecto a las conductas de mobbing se relaciona positivamente e influye en la aparición de estas.

\section{Potenciales efectos y consecuencias del mobbing}

En cuanto a los efectos del acoso laboral, se han propuesto modelos que inciden en aspectos personales de bienestar y salud (Moreno et al., 2007), mientras que otros lo hacen en aspectos organizacionales, tales como satisfacción laboral y compromiso organizacional (Salin, 2003). También se encuentran estudios que no han encontrado relaciones significativas entre acoso y bienestar psicológico, problemas de salud físicos o de satisfacción laboral (Topa et al., 2007).

En lo referente a la salud y las bajas por enfermedad, en cuanto potencial consecuencia del acoso, un reciente metaanálisis encuentra relaciones positivas moderadas entre acoso y absentismo por motivos de salud en 16 de los 17 estudios analizados (Nielsen et al., 2016). Con el fin de seguir profundizando en las posibles relaciones entre el mobbing y la percepción de salud, se propone la siguiente hipótesis:

- H10: las conductas de mobbing influyen la percepción de salud según encuestas autoinforme.

\section{Método}

\section{Muestra}

La muestra final la componen 103 participantes $(N=$ $103)$, de los cuales el $51.46 \%$ son varones y el $48,54 \%$ mujeres, pertenecientes a la población española en activo, principalmente de la Comunidad Valenciana $(83,5 \%)$. Los encuestados están empleados en distintas organizaciones del sector educativo. Trabaja a tiempo completo el $85 \%$ y a tiempo parcial el $14 \%$.

La edad media es de 45.83 años (DT $=9.21$ ). El $82.52 \%$ ha completado estudios universitarios y un $17.48 \%$ declaran haber realizado otro tipo de estudios. La antigüedad media en la empresa actual es de 14.86 años (DT = 10.67).

El $82.52 \%$ desempeña su labor profesional en la empresa pública, frente a un $14.56 \%$ que lo hace en la privada y un $2.91 \%$ que lo hace en la empresa privada con capital público. En lo relativo a la categoría profesional, el $68 \%$ se declara empleado de su organización, el $14 \%$ mando intermedio, el $9 \%$ directivos, el $5 \%$ 
propietario o alto directivo y un $4 \%$ se declara en otras posiciones.

El $73 \%$ forma parte de empresas con más de 200 empleados, aspecto previsible dado que la búsqueda de participantes se ha realizado, principalmente, en universidades públicas, frente a un $12 \%$ que lo hace en empresas de 10 a 49 empleados, un $8 \%$ en empresas de 50 a 199 empleados y el $7 \%$ restante en microempresas de 0 a 9 empleados. El $49 \%$ de los encuestados declara no tener personal a su cargo, frente al $51 \%$ que declara tener subordinados.

\section{Variables e instrumentos}

Las variables del presente estudio se han medido mediante un cuestionario autoinformado que contenía los siguientes bloques y escalas:

- Perfil sociolaboral de los participantes. Características demográficas (género, edad y estudios) y características laborales (antigüedad, categoría, dependientes, situación laboral/tipo de contrato).

- Perfil organizacional. Tipo de empresa (titularidad del capital) y tamaño en número de empleados.

- Negative Acts Questionnaire (NAQ) (Einarsen y Raknes, 1997). De este cuestionario se ha empleado una versión reducida adaptada a la población española (NAQ-RE) (Moreno et al., 2007). La forman 14 ítems referidos a conductas negativas que han podido sufrir los encuestados. Esta escala se caracteriza por evitar la etiqueta de acoso para centrase en la descripción de la frecuencia con la que se es víctima de determinadas acciones por parte de otros (Topa et al., 2009). Emplea una escala de respuesta tipo Likert de cinco puntos que abarca desde 1 (nunca) a 5 (diariamente). La escala carga en dos factores relacionados: acoso relacionado con el trabajo y acoso dirigido a la persona (Moreno et al., 2007; Topa et al., 2009). Se ha empleado con el propósito de medir las posibles conductas de acoso, entendidas como la variable criterio de este estudio. En la muestra en estudio la escala completa ha arrojado una fiabilidad (alfa de Cronbach) $\alpha=0.94$, para la subescala de acoso relacionado con el trabajo $\alpha=$ 0.92 y para la subescala de acoso relacionado con la persona $\alpha=0.89$.

- Escala de Tolerancia Organizacional al acoso Psicológico en el Trabajo-OT (Pérez-Larrazabal, 2016). Esta se evaluó recientemente en un estudio exploratorio y confirmatorio de Pérez-Larrazabal et al. (2019). En el presente estudio se emplea a fin de medir la tolerancia al acoso en la organización como una posible variable predictora de mobbing. La escala de respues- tas es tipo Likert de cinco niveles y comprende desde 1 (muy en desacuerdo) hasta 5 (muy de acuerdo). La componen 18 ítems. El índice de fiabilidad obtenido para la muestra de este estudio ha sido $\alpha=0.87$. Los análisis factoriales realizados por el autor y sus colegas indican la existencia de cinco factores: formación, evaluación, coherencia, ética y promoción. En el presente estudio se ha realizado un análisis factorial confirmatorio en el que se identifican las cinco dimensiones [ $\chi^{2}(242.695)$; d. f. $\left.=94 ; p<0.001\right]$, si bien no se observa un buen ajuste del modelo propuesto a los datos $(\mathrm{CFI}=0.87$; RMSEA $=0.124)$. La estructura factorial de la escala OT para los datos de la muestra se presenta en la Figura 1.

Figura 1. Estructura factorial de la escala de tolerancia organizacional para los datos de la muestra

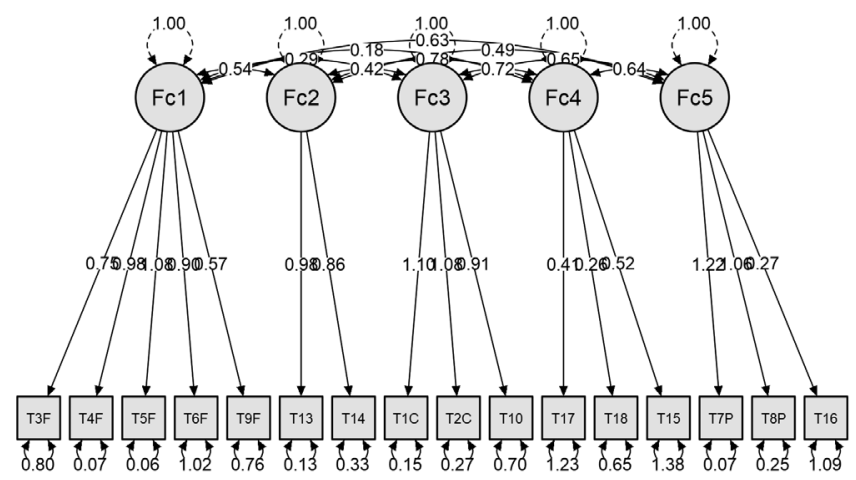

En la muestra de este estudio se han obtenido los siguientes valores de fiabilidad para las cinco subescalas: $\alpha=0.80$ con respecto al factor formación; $\alpha=0.85$ para el factor evaluación; $\alpha=0.89$ para el factor coherencia; $\alpha=0.27$ para el factor ética y $\alpha=0.73$ para el factor promoción.

- Escala de Identificación con la Profesión, la Organización y el Grupo de Trabajo. Empleada con la finalidad de medir la identificación como otra de las posibles variables predictoras. La forman tres subescalas de cuatro ítems, extraídos de la Escala de identificación Organizacional-OID (Mael y Ashforth, 1992). La escala completa obtiene una fiabilidad para la muestra $\alpha=0.93$. Las tres subescalas propuestas son: a) subescala de identificación con la profesión, formada por cuatro ítems con una fiabilidad para esta muestra $\alpha=0.78$; b) subescala de identificación con el grupo de trabajo, formada por cuatro ítems y con una fiabilidad $\alpha=0.86$, y c) subescala de identificación con la organización, formada también por cuatro ítems con una fiabilidad de 0.86 . Las tres subescalas son tipo Likert y van desde 1 (muy en desacuerdo), hasta 5 (muy de acuerdo). 
- Escala de Autoevaluación de Adaptabilidad. Está basada en taxonomía de ocho dimensiones de rendimiento adaptativo que definen Pulakos et al. (2000, p. 617). La conforman 15 ítems tipo Likert que comprenden desde 1 (nunca) a 5 (siempre), con una fiabilidad $\alpha=0.93$ para la muestra en estudio.

- Escala Subjetiva de Quejas de salud. Se emplea como medida de la autopercepción de salud. Está basada en The Subjective Health Complaint Inventory (SHC) (Eriksen et al., 1999), compuesta por 22 ítems tipo Likert que van desde 1 (nunca) a 5 (todo el tiempo), con una fiabilidad de 0.80 .

\section{Procedimiento}

El cuestionario se distribuyó en línea mediante Google Forms $^{\circledR}$. Se incorporaron leyendas en el encabezado para informar a los participantes de los objetivos generales de la investigación, de la necesidad de responder con sinceridad a los diferentes ítems y de la preservación del anonimato, así como de la confidencialidad absoluta de los datos facilitados. A fin de evitar la omisión de preguntas se empleó la condición "necesidad de responder todas las preguntas" para poder grabar el cuestionario.

Se enviaron 630 correos electrónicos a diversas instituciones universitarias españolas, principalmente de Valencia y Barcelona, y a otras entidades no universitarias en las que se imparte formación. La aceptación de cuestionarios se extendió durante tres meses. El índice de respuesta ha sido ligeramente inferior al $16 \%$.

Los análisis estadísticos de los resultados se han realizado con Excel y JASP (0.11.1). Se ha comprobado la normalidad de los datos aplicando la prueba $Z$ de Kolmogorov-Smirnov. Los datos de las escalas de acoso (NAQ) y de identificación en sus tres dimensiones (profesión, organización y grupo) no presentan una distribución normal, por lo que se ha optado por realizar análisis no paramétricos.

\section{Resultados}

A fin de analizar la incidencia de acoso en la muestra con la escala (NAQ-RE) se ha calculado la puntuación total de cada sujeto, así como los estadísticos descriptivos. En la interpretación del nivel de acoso se ha seguido el criterio de la suma de las puntuaciones de los 24 ítems con cinco puntos de corte (García-Izquierdo et al., 2004): se descarta la existencia de riesgo de mobbing para puntuaciones inferiores a 29 puntos, se considera un riesgo bajo para puntuaciones entre 29 y 32 puntos, nivel medio para puntuaciones entre 33 y 36 y nivel alto entre 37 y 41; para puntuaciones por encima de 42 se considera un nivel de riesgo muy alto de acoso. Debido a que la escala empleada en este estudio ha sido la versión reducida de 14 elementos (NAQ-RE), se han calculado las correspondencias adaptadas a esta puntuación, de modo que queda el criterio, tal como se indica en la Tabla 1.

Tabla 1. Criterios de interpretación de la escala NAQ-RE e incidencia en la muestra (\%)

\begin{tabular}{lccccc}
\hline & \multicolumn{5}{c}{ Niveles de riesgo de acoso } \\
\hline & Nulo & Bajo & Medio & Alto & Muy alto \\
\hline $\begin{array}{l}\text { Puntuación } \\
\text { criterio }\end{array}$ & $<17$ & $17-19$ & $20-21$ & $22-24$ & $>24$ \\
\hline \begin{tabular}{l} 
\% en la muestra \\
\hline
\end{tabular} & 21 & 23 & 5 & 13 & 38 \\
\hline
\end{tabular}

La escala de acoso (NAQ-ER) presenta un valor total medio por encuestado de 24.61 (DT=10.09), con un valor mínimo de 14 puntos y un máximo de 59; la mediana se sitúa en 22 puntos. Según el baremo empleado en la Tabla 1 y las frecuencias observadas en la muestra, el $79 \%$ de los encuestados informa de riesgo de acoso, de manera que es un riesgo de alto a muy alto para el $51 \%$ de los participantes. Se han conformado los distintos grupos por riesgo, y son el grupo 1 el de riesgo nulo y el grupo 5 el de máximo riesgo.

Respecto a las posibles relaciones del acoso con las distintas variables que se han propuesto en el estudio y al aplicar los criterios de interpretación de Sancho et al. (2014), se encuentran correlaciones significativas de nivel medio-bajo entre la escala de acoso (NAQ-RE) y las variables que se muestran en la Tabla 2.

En cuanto a la posible relación entre las variables planteadas como potenciales predictores se encuentra correlación significativa entre la escala de tolerancia organizacional al mobbing (OT) y la escala de identificación completa $(0.26 ; \mathrm{p}<0.01)$ que se fortalece cuando la relación se centra en la subescala de identificación con la organización $(0.36 ; \mathrm{p}<0.01)$. Igualmente, se observa correlación con la escala de identificación con el grupo $(0.23 ; p<0.05)$. Existe correlación entre identificación $\mathrm{y}$ adaptabilidad $(0.23 ; \mathrm{p}<0.05)$, concretamente la correlación se da entre adaptabilidad e identificación con el grupo $(0.30 ; \mathrm{p}<0.01)$ e identificación con la organización $(0.21 ; \mathrm{p}<0.05)$.

Los resultados con respecto a la incidencia por sexos indican que las mujeres puntúan ligeramente superior en la escala de acoso que los hombres, con una puntuación media de $26.44(\mathrm{DT}=10.55)$ y de 22.89 (DT $=9.41)$, respectivamente. La prueba Mann-Whitney indica una diferencia significativa en las medias de acoso que informan 
ambos grupos $(U=1056 ; p<0.05)$. No se observan diferencias significativas en las dos subescalas por separado.

Tabla 2. Caracterización de las variables de estudio y correlaciones

\begin{tabular}{|c|c|c|c|}
\hline & Media & DT & NAQ-RE \\
\hline NAQ-RE & 1.76 & 0.72 & 1 \\
\hline $\begin{array}{l}\text { NAQ-RE-acoso relacionado } \\
\text { con el trabajo }\end{array}$ & 1.85 & 0.77 & --- \\
\hline $\begin{array}{l}\text { NAQ-RE-acoso relacionado } \\
\text { con la persona }\end{array}$ & 1.64 & 0.74 & --- \\
\hline Edad & 45.83 & 9.21 & $-0.25^{*}$ \\
\hline Nivel de estudios & --- & ---- & $-0.38^{* * *}$ \\
\hline Tamaño de la empresa & --- & --- & $-0.29 * *$ \\
\hline Escala tolerancia $(\mathrm{OT})$ & 2.37 & 0.56 & $-0.33 * * *$ \\
\hline OT-Formación & 2.56 & 0.73 & $-0.38^{* * *}$ \\
\hline OT-Evaluación & 2.52 & 0.40 & $-0.29 * *$ \\
\hline OT-Coherencia & 2.40 & 0.47 & $-0.22 *$ \\
\hline OT-Ética & 2.09 & 0.15 & -0.02 \\
\hline OT-Promoción & 2.10 & 0.86 & -0.15 \\
\hline IDENTIFICACIÓN & 3.46 & 0.25 & -0.19 \\
\hline $\begin{array}{l}\text { Identificación con } \\
\text { la profesión }\end{array}$ & 3.52 & 0.29 & -0.09 \\
\hline $\begin{array}{l}\text { Identificación con } \\
\text { la organización }\end{array}$ & 3.30 & 0.18 & $-0.21^{*}$ \\
\hline Identificación con el grupo & 3.57 & 0.22 & $-0.20^{*}$ \\
\hline Adaptabilidad & 3.97 & 0.20 & $-0.25^{* *}$ \\
\hline Quejas de salud & 2.18 & 0.68 & $0.35^{* * *}$ \\
\hline
\end{tabular}

En lo relativo a las frecuencias por sexo y grupo de riesgo, los resultados se muestran en la Tabla 3.

Tabla 3. Incidencia (\%) de acoso por grupo de riesgo y sexo

\begin{tabular}{cccccc}
\hline \multicolumn{5}{c}{ Riesgo de acoso } \\
\hline Sexo & Nulo & Bajo & Medio & Alto & Muy alto \\
\hline Hombres & 24.53 & 30.19 & 0 & 15.09 & 30.19 \\
\hline Mujeres & 18 & 14 & 10 & 10 & 48 \\
\hline
\end{tabular}

Con la finalidad de analizar la correlación negativa observada respecto a la edad de los encuestados, se han establecido tres grupos de edad: grupo 1 (21-35), grupo 2 (36-50), grupo $3(51-65)$ y se ha puesto a prueba la diferencia de medias entre los tres grupos. El análisis descriptivo muestra diferencias en las puntuaciones medias de acoso por edad: grupo $1(2.16 ; \mathrm{DT}=0.72)$, grupo $2(1.77 ; \mathrm{DT}=0.72)$ y grupo $3(1.56 ; \mathrm{DT}=0.67)$. El resultado de la prueba Kruskal-Wallis, para estimar si dichas diferencias son sig- nificativas ha sido [H (8.05); d. f. $=2 ; p<0.05]$. En el análisis de subescalas, se encuentra una diferencia significativa en las medias de acoso relacionado con el trabajo entre los tres grupos [H (7.72); d. f. $=2 ; p<0.05]$.

En cuanto a las frecuencias por grupo de riesgo y edades, los resultados se muestran en la Tabla 4.

Tabla 4. Incidencia de acoso \% (N) por grupo de riesgo y edad

\begin{tabular}{lccccc}
\hline \multicolumn{5}{c}{ Riesgo de acoso } \\
\hline Edad & Nulo & Bajo & Medio & Alto & Muy alto \\
\hline $21-35$ & $0(0)$ & $4.35(1)$ & $20(1)$ & $7.69(1)$ & $27.50(11)$ \\
\hline $36-50$ & $59.09(13)$ & $69.57(16)$ & $20(1)$ & $23.08(3)$ & $62.50(25)$ \\
\hline$>51$ & $40.91(9)$ & $26.09(6)$ & $60(3)$ & $69.23(9)$ & $10(4)$ \\
\hline
\end{tabular}

No se ha observado correlación significativa entre la variable acoso y la antigüedad del empleado (-0.13; $p>0.05)$.

En lo relativo al nivel de estudios se observa una correlación negativa significativa (Tabla 2). Los valores medios en las puntuaciones en la escala NAQ-RE de los diferentes grupos han sido: bachillerato $(2.33$; DT $=$ $0.66)$, universitarios $(1.83$; DT $=0.74)$ y otros estudios superiores $(1.23$; DT $=.29)$. Se han puesto a prueba las diferencias de medias por grupos según niveles de estudios y los resultados son significativos [H (14.93); d. f. $=2 ; p<0.001]$. Por tipo de acoso, se encuentra que en las puntuaciones de acoso relacionado con el trabajo existen diferencias significativas según tipos de estudios [H (15.52); d. f. $=3 ; p<0.001]$ y también en la escala de acoso relacionado con la persona $[\mathrm{H}(8.93)$; d. f. $=3$; $p<0.05]$.

En lo que atañe a variables organizacionales se ha planteado la relación entre el tamaño de la organización y la incidencia de acoso. Se observa una correlación media-baja de signo negativo [-0.29; $\mathrm{p}<0.01]$, lo cual indica que a mayor número de empleados se producen menos conductas de mobbing.

Se ha observado una puntuación media mayor en la escala de acoso en empresas de tamaño medio, mientras que en las empresas de más de 200 empleados el riesgo de acoso aparentemente es menor. La prueba KruskalWallis confirma que existe una diferencia significativa entre los distintos grupos analizados [H (14.86); d. f. $=3$; $p<0.001]$.

Al objeto de analizar posibles relaciones causales y posibles variables mediadoras entre las variables propuestas, se ha llevado a cabo un análisis SME de mediación, aplicando el método Delta de errores estándar y estimador ML. 
Los efectos directos de la relación acoso-salud [ES = $0.60 ; \mathrm{DT}=0.13 ; p<0.001]$ se reducen al introducir la variable tolerancia organizacional como mediadora de tal relación. El efecto indirecto del acoso pasa a ser mínimo y no significativo [ $\mathrm{ES}=-2.28 \mathrm{e}^{-3}$; DT $=0.04 ; p>0.05$ ].

Al poner a prueba las variables que se postulan en las hipótesis 1 a 4 y 7, según el análisis de efectos directos, se confirma el género como variable antecedente del acoso [ES $=0.40 ; \mathrm{DT}=0.18 ; p<0.05]$, el nivel de estudios $[\mathrm{ES}=-0.57 ; \mathrm{DT}=0.20 ; \mathrm{p}<0.01]$ y el tamaño de la organización [ES $=-0.24 ; \mathrm{DT}=0.10 ; p<0.05]$.

No se confirman como antecedentes la edad o la antigüedad en la organización. Sin embargo, al introducir estas variables como posibles factores de confusión entre la relación acoso-tolerancia-salud, se reducen los efectos directos del acoso en la salud [ES $=0.47$; DT $=0.09$; $p<0.001$ ], mientras que los efectos de amortiguación de la tolerancia organizacional se mantienen.

Si se considera la tolerancia organizacional como variable antecedente del acoso, se observa un efecto directo de carácter negativo $[\mathrm{ES}=-0.54 ; \mathrm{DT}=0.15$; $p<0.001]$.

Al considerar la adaptabilidad como posible variable mediadora, los efectos indirectos del acoso pasan a ser no significativos [ES $=0.02 ; \mathrm{DT}=0.02 ; p>0.05$ ] Siguiendo el mismo proceso con la variable identificación, se observa igual reducción de los efectos indirectos del acoso [ES $\left.=-1.18 \mathrm{e}^{-3} ; \mathrm{DT}=8.81 \mathrm{e}^{-3} ; p>0.05\right]$.

El modelo multivariante que contempla como variables mediadoras simultáneas la identificación, la adaptabilidad y la tolerancia organizacional indica que los efectos totales directos del acoso sobre la salud son significativos de grado medio [ES $=0.60 ; \mathrm{DT}=0.12 ; p<0.001]$, mientras que los indirectos con la mediación de las variables citadas pasan a ser no significativos [ES $=0.05 ; \mathrm{DT}=0.06 ; p>0.05$ ] El modelo explica un $25 \%$ de la varianza en la variable acoso. El gráfico del modelo se presenta en la Figura 2.

No se observan efectos directos significativos en el acoso si se consideran como predictoras las variables adaptabilidad e identificación. Si se analizan por separado las dimensiones de la identificación y de la variable acoso, se observa el mayor efecto indirecto en la relación identificación con la organización-tolerancia organizacional-acoso relacionado con el trabajo $[\mathrm{ES}=-0.20$; $\mathrm{DT}=0.06 ; p<0.001]$.

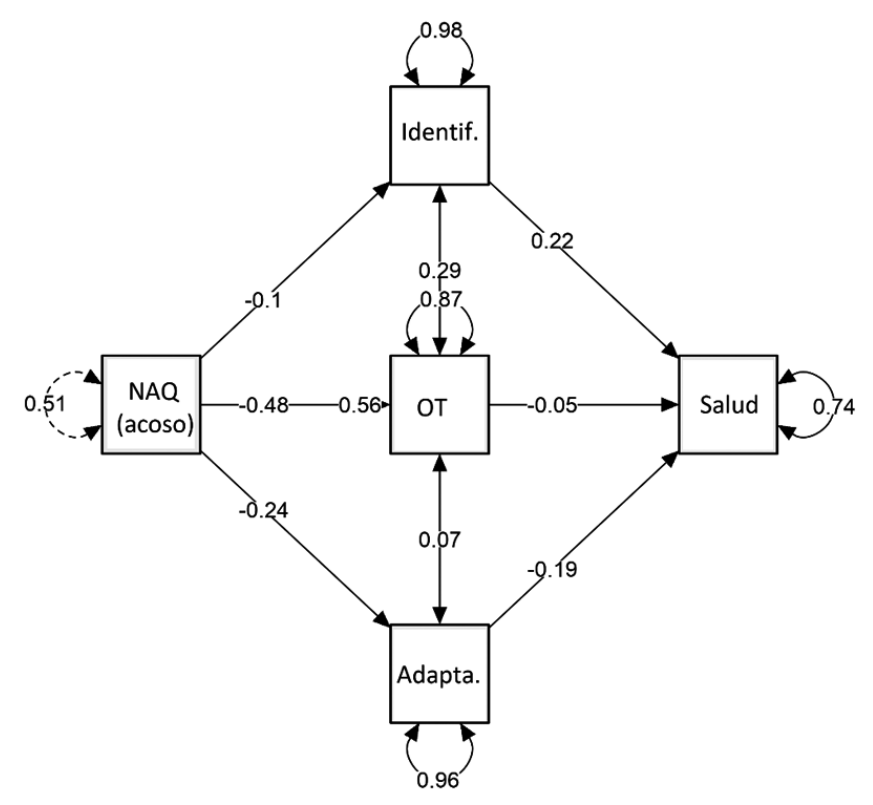

\section{Discusión}

El mobbing o acoso psicológico en el trabajo ha acaparado el interés investigador de las tres últimas décadas, si bien, a pesar de estos esfuerzos por comprenderlo, detectarlo y controlarlo, resulta complicado conocer bien su incidencia en el ámbito laboral a nivel general y por sectores de actividad. A esto se suma la limitada evidencia empírica para algunas de las variables que se relacionan con él y la controversia entre distintos estudios (Topa et al., 2007; Pérez-Larrazábal et al., 2019). Sin embargo, hay consenso en que el acoso representa un problema creciente del ámbito laboral con efectos nocivos tanto para las personas como para el rendimiento de las organizaciones (Einarsen et al., 2003; Cortina, 2008; Hirigoyen, 1998; Leymann, 1996).

Desde una perspectiva psicosocial, este estudio ha tratado de profundizar en el conocimiento de este fenómeno multidimensional y multicausal de los ambientes laborales (Zapf, 1999). La investigación se ha centrado en el sector educativo, en una muestra de 103 profesionales españoles del sector educativo en activo. Se les ha invitado a participar en un cuestionario autoinformado compuesto por diversas escalas de variables relacionadas con el acoso en la literatura. El cuestionario se administró en línea y la muestra se captó en un periodo de tres meses, mediante 630 correos electrónicos enviados a los directorios de diferentes instituciones educativas españolas. 
Sobre la base de los resultados obtenidos, el primer dato a destacar es la alta incidencia de mobbing dentro de la muestra estudiada. Empleando el criterio sumatorio de García-Izquierdo et al. (2004) adaptado al número de ítems de la escala reducida NAQ-RE, se observa una alta puntuación media $(24.61$; DT $=10.09)$ en el total de ítems de la escala de acoso. El 79 \% de los encuestados informa que ha sufrido algún tipo de acoso y solo el $21 \%$ no se ha sentido acosado en su entorno de trabajo. El $56 \%$ muestra un nivel de acoso de medio a alto y el $38 \%$ declara estar sometido a un nivel muy elevado de acoso. Estas cifras se sitúan bastante por encima de las que aparecen en la Sexta Encuesta Europea sobre Condiciones de Trabajo-2015 (Parent-Thirion et al., 2016), la cual estima una incidencia del $10 \%$ para la población española. Tal vez en este notable aumento de la incidencia de acoso haya influido la situación económica y laboral, así como los recortes sobrevenidos en el sector educativo durante la última década a causa de la crisis económica del 2008. Llama la atención, asimismo, la elevada incidencia en un sector de alta cualificación, dado que la mayoría de los encuestados son docentes, cuando el mobbing se creía más asociado a sectores con una menor cualificación (Meseguer de Pedro et. al. 2007; López-Cabarcos et al., 2010).

En la línea de trabajos anteriores (Topa et al. 2007), se ha encontrado que la población femenina padece más este fenómeno, con lo que se confirma un efecto directo entre el género y la incidencia de acoso, lo que respalda la primera hipótesis (H1) de este estudio.

Se confirma que los jóvenes son, con mayor frecuencia, víctimas de acoso en comparación con los empleados de mayor edad, lo que da soporte a la segunda hipótesis $(\mathrm{H} 2)$, si bien no se ha observado efectos directos ni mediados entre la variable edad y la escala de acoso. En este aspecto es necesario destacar que la muestra de jóvenes es más reducida que el resto de edades, por lo que sería conveniente continuar el estudio ampliando la muestra en este rango de edad a fin de comprobar si las diferencias en puntuación de acoso por edades se mantienen.

No se ha encontrado relación entre la antigüedad laboral y las conductas de acoso, por lo que la hipótesis H3 no se sostiene. Como se planteaba en la hipótesis H4, el nivel formativo correlaciona de forma negativa con el riesgo de acoso $(-0.38 ; \mathrm{p}<0.001)$. También se observa un efecto directo negativo entre el nivel formativo y la puntuación en la escala de acoso. Podría plantearse que a mayor nivel formativo, mayor nivel de independencia jerárquica, lo cual podría prevenir del riesgo de acoso descendente $\mathrm{y}$, tal vez, horizontal.

Con respecto a variables relacionadas con la conducta del individuo, se comprueba que la capacidad de adaptación del empleado correlaciona negativamente con las conductas de acoso percibidas $(-0.25 ; p<0.01)$, lo que da soporte a la hipótesis H5. Se comprueba, también, que correlaciona positivamente con la identificación del individuo con el grupo $(0.30 ; p<0.01)$ y con la organización $(.21 ; p<0.05)$, lo cual parece cobrar lógica. Se ha propuesto que la adaptabilidad sería un regulador o freno de las conductas de acoso (Pérez-Larrazabal, 2016). Efectivamente, en esta muestra se observan efectos de mediación de esta variable en las relaciones del género, el nivel de estudios y el tamaño de empresa con la variable acoso.

Se han encontrado relaciones negativas entre el acoso y la identificación del individuo con la organización $(-0.21 ; p<0.05)$ y con el grupo de trabajo $(-0.20 ; p$ $<0.05$ ), lo que da respaldo parcial a la hipótesis H6, pues no se ha encontrado relación significativa entre el acoso y la identificación con la profesión. Se han observado efectos mediadores de la variable identificación entre las variables antecedentes de este estudio y el acoso como variable dependiente. Por otra parte, al estar la antigüedad relacionada con la identificación en el entorno de trabajo, debería profundizarse más en la posible relación entre antigüedad y acoso, de modo que se analice no solo la antigüedad en la organización, sino, también, en el grupo de trabajo y en la profesión.

Se ha abordado, asimismo, un aspecto relacionado con la estructura de la organización, como lo es el número de empleados. En contra de lo afirmado en estudios previos (Einarsen, 2000; Einarsen et al., 2003), se ha encontrado una correlación negativa entre el tamaño y el acoso $(-0.29 ; p<0.01)$, es decir, la incidencia de acoso desciende según aumenta la plantilla. Además, en el analisis de mediación se encuentra un efecto directo negativo de la variable tamaño en la variable acoso. Esto confirma la hipótesis $\mathrm{H} 7$ que planteaba una relación entre las variables pero no establecía su sentido. Podría entenderse que organizaciones más grandes tienen mejor reguladas sus políticas de relación interna, lo que puede reducir la incidencia de acoso. Aun así, los mayores índices de acoso se localizan en empresas de mediano tamaño, entre $10 \mathrm{y}$ 200 empleados y, sobre todo, en el rango entre diez y 49 que, por otra parte, son mayoritarias en nuestra actividad económica. En cuanto a las organizaciones de menos de diez empleados, la menor incidencia podría explicarse por la dificultad que encuentra el acosador para encontrar apoyos o pasar desapercibido en un círculo más reducido de personas (Einarsen, 2000). Futuros estudios deberían incidir en este aspecto e intentar determinar si es un fenómeno sectorial o, por el contrario, intersectorial.

En cuanto a la hipótesis H8 que planteaba la posible relación entre la situación de contratación a tiempo parcial y el riesgo de sufrir acoso, en la muestra estudiada no se confirma. 
Desde la óptica de las posibles variables del clima organizacional que se relacionan con el acoso, se ha abordado la tolerancia organizacional. Se ha comprobado una relación negativa entre el acoso y la baja tolerancia tolerancia organizacional en la organización con las conductas de acoso $(-0.33 ; p<0.001)$. Asimismo, se confirma el efecto directo negativo de la tolerancia organizacional sobre las conductas de acoso $[\mathrm{ES}=-0.54$; DT $=-15$; $p<0.001]$. En este sentido, cabe aclarar que la escala OT está formulada en positivo, de manera que a mayor puntuación menor tolerancia al acoso y, por tanto, la escala correlaciona de forma negativa con la escala NAQ-RE, lo que da respaldo al planteamiento de la hipótesis H9. Esto apunta a que una buena regulación de las políticas sobre justicia organizacional, antidiscriminatorias y penalizadoras de las conductas de acoso ayudan a reducir la incidencia del mobbing, tal como lo han indicado anteriores estudios (Cortina, 2008).

Se ha postulado, también, la posible relación de las conductas de acoso con problemas de salud autoinformados, si bien en la literatura se encuentran resultados contradictorios (Topa et al., 2007). A fin de arrojar algo de luz en esta controversia, se ha postulado la hipótesis H10 sobre posibles correlaciones positivas entre el mobbing y la salud percibida por la víctima. Los resultados arrojan una correlación positiva de grado medio entre ambas variables $(0.35, p<0.001)]$. De igual forma, se comprueba un efecto directo de las conductas de acoso sobre la salud autoinformada [ES $=0.60$; DT $=0.13 ; i<$ $0.001]$. Ambos resultados dan apoyo a la hipótesis planteada; además, la relación es más fuerte que la encontrada en estudios anteriores, en los que se situaba en 0.18 (Topa et al., 2007).

En cuanto al estudio de posibles antecedentes y consecuentes en las relaciones analizadas, un primer modelo que contempla las variables género, el nivel de estudios y el tamaño organizacional, e incluye como variables mediadoras la identificación, la adaptabilidad y la tolerancia al acoso. Este modelo explicaría el $27 \%$ de la varianza en la escala de acoso. El mismo resultado se obtiene al incluir la variable tolerancia organizacional como predictora del acoso, en lugar de mediadora.

Los resultados apuntan a que la cultura organizacional influye en el acoso, por lo menos en lo que concierne a la tolerancia con estas conductas incívicas. En este sentido, cabe animar a las organizaciones a mejorar sus políticas antidiscriminatorias y penalizadoras del acoso, así como a fomentar los entornos sanos (Salanova et al., 2019) en los que el respeto y las conductas cívicas sean la norma, como ya lo hicieran anteriores estudios (Cortina, 2008).
Al analizar los posibles consecuentes, se ha empleado como variable criterio la salud, como predictora el acoso y como mediadoras la identificación y la adaptabili$\mathrm{dad}$. Se encuentra que este modelo explica un $25 \%$ de la varianza en la puntuación en salud, comprobándose un efecto directo de la variable acoso sobre la variable salud, tal como se ha comentado. Se observa, por tanto, que las conductas de acoso influyen en el bienestar psicológico y físico de las personas, lo que termina por conducir a absentismo y abandono, tal como lo han denunciado diferentes estamentos relacionados con el trabajo (González, 2002); no obstante, esta situación puede mejorarse en la medida en que se combata la tolerancia organizacional.

Esto reafirma la necesidad de actuar en pro del bien del conjunto de la sociedad, pues los efectos del mobbing afectan tanto a la salud como a la economía de la sociedad en su conjunto, mediante la posible incidencia en el absentismo, el rendimiento y/o las bajas laborales. Los entornos que promueven comportamientos que protegen la salud y el bienestar de los trabajadores terminan por ser, también, los entornos más productivos y sostenibles (Salanova et al., 2019), por lo que no se advierte ventaja alguna en mantener culturas tóxicas y entornos nocivos laborales, ni para el bienestar de las personas y el desarrollo de la organización ni para la sociedad en su conjunto, que es la que, finalmente, termina por asumir los costes sanitarios derivados de estos comportamientos.

Aparte de las posibles líneas futuras de investigación que se han comentado en el texto y mejorarían la comprensión del fenómeno, cabe indicar que este estudio tiene algunas debilidades que deben considerarse al interpretar los resultados obtenidos. La primera está relacionada con el tipo de muestreo; es limitado, no probabilístico y, parcialmente, de conveniencia. La segunda tiene relación con el carácter autoinformado de los datos, lo que se relaciona con un déficit de validez debido al sesgo de autoinforme, por lo que sería aconsejable buscar la triangulación de los datos en futuros estudios (por ejemplo, al aplicar técnicas cualitativas). La tercera tiene que ver con los instrumentos empleados y el propio diseño que invitan a interpretar con prudencia posibles relaciones causales aquí expuestas que deben ponerse a prueba con diseños experimentales.

A pesar de estas debilidades, el estudio permite conocer más a fondo, en el sector educativo, algunas de las relaciones propuestas en la literatura y sus potenciales efectos, así como introducir variables más recientes como la tolerancia organizacional, la cual se muestra clave a fin de comprender mejor este perjudicial fenómeno de los entornos laborales actuales y desarrollar políticas para erradicarlo. 


\section{Referencias}

Andersson, L. M., \& Pearson, C. M. (1999). Tit for tat? The spiraling effect of incivility in the workplace. Academy of Management Review, 24(3), 452-471.

Bandura, A., Ross, D., \& Ross, S. (1963). Imitation of film-mediated agressive models. Journal of Abnormal and Social Psychology, 66, 3-11.

Baron, R. A., \& Richardson, D. R. (1994). Perspectives in social psychology: Human aggression ( $2^{\mathrm{a}}$ ed.). Plenum Press.

Björkqvist, K., Österman, K. , \& Hjelt-Bäck, M. (1994). Aggression among University Employees. Aggressive Behavior, 20(3), 173-184.

Cortina, L. M. (2008). Unseen injustice: Incivility as modern discrimination in organizations. Academy of Management Review, 33(1), 55-75.

Cortina, L. M. (2015). Workplace harassment: Examining the scope of the problem and potential solutions. En Proceedings of the Select Task Force Meeting (June 15, 2015). https://www.eeoc.gov/written-testimony-lilia-m-cortina-phd-professor-psychologyand-womens-studies-university-michigan

Cortina, L. M. (2016). Rebooting workplace harassment prevention (written testimony of Lilia Cortina. Meeting of June 20, 2016). Equal Employment Opportunity Commission.

Einarsen, S. (1999). The nature and causes of bullying at work. International Journal of Mapower, 20(1/2), 16-27.

Einarsen, S. (2000). Harassment and bullying at work: A review of the scandinavian approach. Aggresion and Violent Behavior, 5(4), 379-401.

Einarsen, S., Hoel, H., Zapf, D., \& Cooper, C. L. (2003). Bullying and emotional abuse in the workplace. Taylor \& Francis e-Library.

Eriksen, H., Ihlebaek, C., \& Ursin, H. (1999). A scoring system for subjective health complaints (SHC). Scandinavian Journal of Public Health, 27, 63.

Einarsen, S., \& Raknes, B. (1997). Harassment at work and victimization of men. Violence and Victims, 12, 247-263.

Farber, B. A., \& Miller, J. (1981). Teacher burnout: A psycho-educational perspective. Teacher College Record, 83(2), 235-243.

Fornés, J., Martínez-Abascal, M. Á., \& García de la Banda, G. (2008). Análisis factorial del cuestionario de hostigamiento psicológico en el trabajo en profesionales de enfermería. International Journal of Clinical and Health Psychology, 8(1), 267-283.

García-Izquierdo, M., Llor, B., Ruiz, J.A., Sáez, M.C., Blasco, J. R., \& Campillo, M. J. (2004). Evaluación del acoso psicológico en el trabajo: El NAQ-RE, revisión de la adaptación española. VII European Conference on Psychological Assesment.

González, K. (2002). Acción sindical y preventiva contra los riesgos psicosociales. El mobbing. Lan Harremanak, 7(II), 183-194.

Hirigoyen, M.F. (1998). Le harcèlement moral. Éditions La Découverte y Syros.

Leymann, H. (1990). Mobbing and psychological terror at workplaces. Violence and Victims, 5(2), 119-126.

Leymann, H. (1996). The content and development of mobbing at work. European Journal of Work and Organizational Psychology, 5(2), 165-184.

López-Cabarcos, M. Á., Vázquez-Rodríguez, P., \& Montes-Piñeiro, C. (2010). Mobbing:Antecedentes psicosociales y consecuencias sobre la satisfacción laboral. Revista Latinoamericana de Psicología, 42(2), 215-224.

Mael, F., \& Ashforth, B. E. (1992). Alumni and their alma mater: A partial test of the reformulated model of organizational identification. Journal of Organizational Behavior, 13, 103-123.

Meseguer de Pedro, M., Soler Sánchez, M. I., Sáez Navarro, M. C., \& García Izquierdo, M. (2007). Incidencia, componentes y origen del mobbing en el trabajo en el sector hortofrutícola. Anales de Psicología, 23(1), 92-100.

Moreno, B., Rodríguez, A., Martínez, M., \& Gálvez, M. (2007). Assessing workplace bullying: Spanish validation of a reduced version of the negative acts questionnaire. The Spanish Journal of Psychology, 10(2), 449-457.

Nielsen, M. B., Indregard, A. M. R, Overland, S. (2016). Workplace bullying and sickness absence: A systematic review and meta-analysis of the research literature. Scandinavian Journal of Work, Environment \& Health, 42(5), 359-370. 10.5271/sjweh.3579

Nielsen M. B., Tvedt S. D., \& Matthiesen S. B. (2013). Prevalence and occupational predictors of psychological distress in the offshore petroleum industry: A prospective study. International Archives of Occupational Environmental Health, 86, 875-85.

Padilla, A., Hogan, R., \& Kaiser, R.B. (2007). The toxic triangle: Destructive leaders, susceptible followers and conducive environments. The Leadership Quartely, 18, 176-194.

Parent-Thirion, A., Biletta, I., Cabrita, J., Vargas-Llave, O., Vermeylen, G., Wilczyńska, A., \& Wilkens, M. (2016). The sixth European Working Conditions Survey (EWCS)-2015. Eurofound. 10.2806/422172

Perez-Larrazabal, J. (2016). Tolerancia organizacional al acoso psicológico en el trabajo: 
desarrollo, validación y estudio empírico de sus efectos en la salud y desempeño laboral (tesis doctoral). Universisdad Nacional de Educación a Distancia.

Perez-Larrazabal, J., \& Topa, G. (2016). Cuando la organización mira hacia otro lado. Tolerancia organizacional. Dyna Management. 4(1), 1-11 http://dx.doi. org/10.6036/MN7857

Perez-Larrazabal, J., Lopez de la Llave, A., \& Topa, G. (2019). Organizational tolerance for workplace harassment: Development and validation of the POT Scale. Sustainability, 11, 4078. 10.3390/su11154078.

Pulakos, E., Donovan, M., \& Plamondon, K. (2000). Adaptability in the workplace: Development of a taxonomy of adaptative performance. Journal of Applied Psychology, 85, 612-624.

Rayner, C. , \& Cooper, C. (1997). Workplace bullying: Myth or reality-can we afford to ignore it? Leadership and Organization Development Journal, 18, 211-214.

Richman, J. A., Rospenda, K. M., Flaherty, J. A., Freels, S., \& Zlatoper, K. (2004). Perceived organizational tolerance for workplace harassment and distress and drinking over time [harassment and mental health]. Women \& Health, 40(4). 10.1300/J013v40n04_01.

Salanova, M., Llorens, S., \& Martínez, I. (2019). Organizaciones saludables: una mirada desde la psicología positiva. Aranzadi.

Salin, D. (2003). Ways of explaining workplace bullying: A review of enabling, motivating and precipitating structures and processes in the work environment. Human Relations, 56(10), 1213-1232.

Sancho, C., González Such, J., \& Bakieva, M. (2014). PSPP. Correlación bivariada. Coeficiente Pearson. Innovamide L4U. Red de Innovación Educativa y Calidad Docente. Universidad de València. http:// www.uv.es/innovamide/14u/PSPP/pspp09/pspp09. wiki

Segurado, A., Agulló, E., Rodríguez, J., Agulló, M. S., Boada, J., \& Medina, R. (2008). Las relaciones interpersonales como fuente de riesgo de acoso laboral en la policía local. Psicothema, 20(4), 739-744.
Tajfel, H. (1970). Experiments in intergroup discrimination. Scientific American, 223, 96-102.

Tajfel, H. (1974). Social identity and intergroup behavior. Social Science Information, 13, 65-93.

Tajfel, H., \& Turner, J. C. (1979). An integrative theory of intergroup conflict. En W. G. Austin y S. Worchel (Eds.), The social psychology of intergroup relations (pp. 33-47). Brooks-Cole.

Topa, G., Depolo, M., \& Morales, J. F. (2007). Acoso laboral: meta-anaálisis y modelo integrador de sus antecedentes y consecuencias. Psicothema, 19(1), 88-94.

Topa G., Moriano J. A., \& Morales F. J. (2008). Identidad social y apoyo percibido en las organizaciones: sus efectos sobre las conductas de ciudadanía. Interamerican Journal of Psychology, 42(2), 363-370.

Topa, G., Moriano, J. A., \& Morales, F. J. (2009). Acoso laboral entre profesionales de enfermería: el papel protector de la identidad social en el trabajo. Anales de Psicología, 25(2), 266-276.

Turner, J. C. (1985). Social categorization and the selfconcept: A social cognitive theory of group behaviour. En E. J. Lawler (Ed.), Advances in group processes: Theory and research (vol. 2, pp. 77-122). JAI Press.

Turner, J. C., \& Brown, R. (1978). Social status, cognitive alternatives and intergroup relations. En $\mathrm{H}$. Tajfel (Ed.), Differentiation between social groups: Studies in the social psychology of intergroup relations. Academic Press.

Turner, J. C., Hogg, M. A., Oakes, P. J., Reicher, S. D., \& Wetherell, M. S. (1987). Rediscovering the social group: A self-categorization theory. Blackwell.

Villagrán, S., Jasso, D., Aldaba, M., \& Rodríguez, M. (2017). Mobbing: impacto psicológico en docentes universitarios, repercusiones en el sentido de pertenencia y permanencia laboral. Pensamiento Americano, 10(18), 77-95.

Zapf, D. (1999). Organisational, work group related and personal causes of mobbing/bullying at work. International Journal of Manpower, 20(1/2), 70-85. 